\title{
REVERSIBLE AND REFLEXIVE PROPERTIES FOR RINGS WITH INVOLUTION
}

\author{
USAMA A. ABURAWASH AND MUHAMMAD SAAD
}

Received 22 September, 2018

\begin{abstract}
In this note, we give a generalization for the class of *-IFP rings. Moreover, we introduce *-reversible and *-reflexive *-rings, which represent the involutive versions of reversible and reflexive rings and expose their properties. Nevertheless, the relation between these rings and those without involution are indicated. Moreover, a nontrivial generalization for *-reflexive *-rings is given. Finally, in *-reversible *-rings it is shown that each nilpotent element is *nilpotent and Köthe's conjecture has a strong affirmative solution.
\end{abstract}

2010 Mathematics Subject Classification: 16W10; 16N60; 16D25

Keywords: involution, quasi-*-IFP, *-reduced, *-nilpotent, *-reversible, *-reflexive and projection *-reflexive

\section{INTRODUCTION}

All rings considered are associative with unity. A $*$-ring $R$ will denote a ring with involution and a self-adjoint ideal $I$ of $R$; that is $I^{*}=I$, is called *-ideal. A projection $e$ of $R$ is an idempotent satisfies $e^{2}=e=e^{*}$. Recall from [7], an idempotent $e \in R$ is left (resp. right) semicentral in $R$ if $e R e=R e$ (resp. $e R e=e R$ ). Equivalently, an idempotent $e \in R$ is left (resp. right) semicentral in $R$ if $e R$ (resp. $R e$ ) is an ideal of $R$. Moreover, if $R$ is semiprime then every left (resp. right) semicentral idempotent is central. A semicentral projection is clearly central. A ring (resp. *-ring) $R$ is said to be Abelian (resp. *-Abelian) if all its idempotents (resp. projections) are central. $R$ is reduced if it has no nonzero nilpotent elements. An involution $*$ is called proper (resp. semiproper) if for every nonzero element $a$ of $R, a a^{*}=0$ (resp. $a R a^{*}=0$ ) implies $a=0$. Obviously, a proper involution is semiproper.

From [5], $R$ is semicommutative or has IFP if the right annihilator $r(a)=\{x \in$ $A \mid a x=0\}$ of every element $a \in R$ is a two-sided ideal. In [1], the involutive version of IFP, that is *-IFP, is given as the ring in which the right annihilator of each element of $R$ is *-ideal. Clearly, each *-ring having *-IFP has also IFP.

Cohn [9] called a ring $R$ reversible (or completely reflexive) if $a b=0$ implies $b a=0$ for every $a, b \in R$. Clearly, the class or reversible rings contains the reduced 
rings. Moreover, each reversible ring has IFP. Moreover, in [9, Theorem 2.2], Cohn proved that for reversible rings, Köthe's conjecture has an affirmative solution. Here, we give a strong affirmative solution for Köthe's conjecture for *-reversible *-rings and show that each nilpotent element is *-nilpotent.

In [13], Mason introduced a generalization of reversible rings; namely reflexive rings. A right ideal $I$ of a ring $R$ is said to be reflexive if $a R b \subseteq I$ implies $b R a \subseteq I$, for every $a, b \in R$. A ring $R$ is called reflexive if 0 is a reflexive ideal. In [10], Kim and Baik defined an idempotent reflexive ideal as a right ideal $I$ satisfying $a R e \subseteq I$ if and only if $e R a \subseteq I$ for $e^{2}=e, a \in R . R$ is an idempotent reflexive ring if 0 is an idempotent reflexive ideal. Obviously, the class of idempotent reflexive rings contains reflexive rings and Abelian rings.

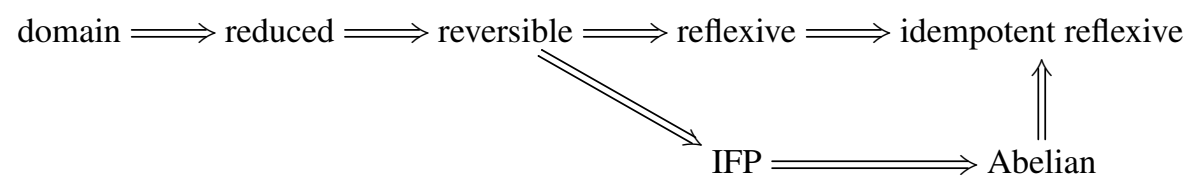

A subring $B$ of a *-ring $R$ is said to be a *-biideal,or self adjoint biideal, of $R$ if $B R B \subseteq B$ and $B^{*}=B$.

Recall from [2], a nonzero element $a$ of a *-ring $R$ is a $*$-zero divisor if $a b=0$ and $a^{*} b=0$ for some nonzero element $b \in R$. Obviously, a *-zero divisor element is a zero divisor, but the converse is not true (example 3 in [2]). A*-ring without *-zero divisors is said to be a $*$-domain.

Recall from [3], an element $a$ of a $*$-ring $R$ is said to be *-nilpotent if there exist two positive integers $m$ and $n$ such that $a^{m}=0$ and $\left(a a^{*}\right)^{n}=0 . R$ is a *-reduced

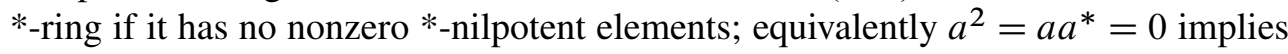
$a=0$ for every $a \in R$. A reduced (or *-domain) *-ring with proper involution is

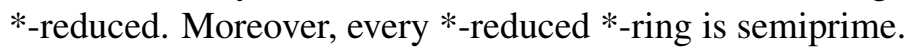

From [4], the *-right annihilator of a nonempty subset $S$ of a *-ring $R$ is the self adjoint biideal $r_{*}(S)=\left\{x \in A \mid S x=0=S x^{*}\right\}$. Finally, $\mathbb{M}_{n}(R)$ will denote the full matrix ring of all $n \times n$ matrices over $R$.

\section{2. *-RINGS WITH QUASI-*-IFP}

In this section, we introduce the property of having quasi- $*$-IFP which generalizes that of having *-IFP introduced in [1].

Definition 1. A *-ring $R$ is said to have quasi-*-IFP if for every $a \in R$, the *-right

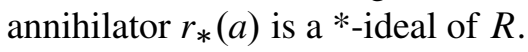

In view of $l_{*}(a)=r_{*}\left(a^{*}\right)$, we see that the *-left annihilator is also *-ideal. Thus the definition of quasi-*-IFP *-ring is left-right symmetric.

Clearly, every *-ring $R$ having *-IFP has also quasi-*-IFP, since $r(a)$ is *-ideal implies $r_{*}(a)=r(a)$ for all $a \in A$. However, the converse is not true as shown by the following example. 
Example 1. Consider the $*$-ring $R=\left(\begin{array}{cc}F & F \\ 0 & F\end{array}\right)$, where $F$ is a field and the adjoint of matrices is the involution. Since $r\left(\left(\begin{array}{ll}1 & 0 \\ 0 & 0\end{array}\right)\right)=\left(\begin{array}{ll}0 & 0 \\ 0 & F\end{array}\right)$ is not an ideal of $R$, then $R$ does not have IFP and consequently does not have *-IFP. Moreover, $R$ has quasi-*-IFP since the *-right annihilator of every nonzero noninvertible element of $R$ takes the form $\left(\begin{array}{cc}0 & F \\ 0 & 0\end{array}\right)$ which is a *-ideal of $R$.

The following are some equivalents for a $*$-ring to have quasi-*-IFP.

Proposition 1. For $a *$-ring $R$, the following conditions are equivalent:

(1) $R$ has quasi-*-IFP.

(2) $r_{*}(S)$ is a *-ideal of $R$ for every subset $S$ of $R$.

(3) $l_{*}(S)$ is $a *$-ideal of $R$ for every subset $S$ of $R$.

(4) For every $a, b \in R, a b=a b^{*}=0$ implies $a R b=0$ (consequently $\left.a R b^{*}=0\right)$

Proof. (1) $\Rightarrow(2)$ : For every $S \subseteq R, r_{*}(S)=\bigcap_{s \in S} r_{*}(s)$ being the intersection of *-ideals is also a $*_{\text {-ideal. }}$

$(2) \Rightarrow(3)$ : From $(2), l_{*}(S)=r_{*}\left(S^{*}\right)$ is a *ideal of $R$.

(3) $\Rightarrow(4): a b=a b^{*}=0$ implies $b^{*} a^{*}=b a^{*}=0$ and consequently $b, b^{*} \in l_{*}\left(a^{*}\right)$ which is a *-ideal of $R$. Hence $b R, b^{*} R \subseteq l_{*}\left(a^{*}\right)$ from which $b R a^{*}=b^{*} R a^{*}=0$ and therefore $a R b=a R b^{*}=0$.

$(4) \Rightarrow(1)$ : Let $x \in r_{*}(a)$, which is a self-adjoint biideal of $R$, then $a x=a x^{*}=0$ implies $a R x=a R x^{*}=0$, form the assumption. Hence $R x \subseteq r_{*}(a)$ which means that $r_{*}(a)$ is a left ideal of $R$. Therefore $r_{*}(a)$ is a *-ideal due to its self-adjointness.

The following results show that quasi-*-IFP implies *-Abelian while the converse is not true.

Proposition 2. Every *-ring with quasi-*-IFP is *-Abelian.

Proof. Let $e$ be a projection in $R$, then $(1-e) e=(1-e) e^{*}=0$ implies $(1-$ $e) R e=0$, from Proposition 1. Hence $e$ is a left semicentral projection and consequently is central.

Moreover, The next example shows that the converse of Proposition 2 is not true.

Example 2. Let $F$ be a field of characteristic 2 and consider the ${ }^{*}$-ring $R=$ $\left\{\left(\begin{array}{cccc}a & a_{12} & a_{13} & a_{14} \\ 0 & a & a_{23} & a_{24} \\ 0 & 0 & a & a_{34} \\ 0 & 0 & 0 & a\end{array}\right) \mid a, a_{i j} \in F\right\}$, with involution defined as 
$\left(\begin{array}{cccc}a & a_{12} & a_{13} & a_{14} \\ 0 & a & a_{23} & a_{24} \\ 0 & 0 & a & a_{34} \\ 0 & 0 & 0 & a\end{array}\right)^{*}=\left(\begin{array}{cccc}a & a_{34} & a_{24} & a_{14} \\ 0 & a & a_{23} & a_{13} \\ 0 & 0 & a & a_{12} \\ 0 & 0 & 0 & a\end{array}\right)$

Since for the matrices $x=\left(\begin{array}{cccc}0 & 1 & -1 & 0 \\ 0 & 0 & 0 & 0 \\ 0 & 0 & 0 & 0 \\ 0 & 0 & 0 & 0\end{array}\right)$ and $y=\left(\begin{array}{cccc}0 & 0 & 0 & 0 \\ 0 & 0 & 0 & 1 \\ 0 & 0 & 0 & 1 \\ 0 & 0 & 0 & 0\end{array}\right)$, we have $x y=0=x y^{*}$, while

$x z y=\left(\begin{array}{cccc}0 & 1 & -1 & 0 \\ 0 & 0 & 0 & 0 \\ 0 & 0 & 0 & 0 \\ 0 & 0 & 0 & 0\end{array}\right)\left(\begin{array}{cccc}a & a_{12} & a_{13} & a_{14} \\ 0 & a & a_{23} & a_{24} \\ 0 & 0 & a & a_{34} \\ 0 & 0 & 0 & a\end{array}\right)\left(\begin{array}{cccc}0 & 0 & 0 & 0 \\ 0 & 0 & 0 & 1 \\ 0 & 0 & 0 & 1 \\ 0 & 0 & 0 & 0\end{array}\right)=$

$\left(\begin{array}{cccc}0 & 0 & 0 & a_{23} \\ 0 & 0 & 0 & 0 \\ 0 & 0 & 0 & 0 \\ 0 & 0 & 0 & 0\end{array}\right) \neq 0$, for every $z \in R$ with $a_{23} \neq 0$, it follows that $R$ does not

have quasi-*-IFP, by Proposition 1 . Moreover $R$ is $*$-Abelian since for any projection $e=\left(\begin{array}{cccc}a & a_{12} & a_{13} & a_{14} \\ 0 & a & a_{23} & a_{24} \\ 0 & 0 & a & a_{34} \\ 0 & 0 & 0 & a\end{array}\right), e^{2}=e^{*}=e$ implies $a_{11}=a_{12}=a_{13}=a_{21}=$ $a_{22}=a_{33}=0$ and $a^{2}=a$, so that $R$ has no nontrivial projections.

Next, we answer the question of when a $*$-ring with quasi-*-IFP is *-reduced.

Proposition 3. Let $R$ be a semiprime *-ring having quasi-*-IFP, then $R$ is $*_{-}$ reduced.

Proof. Let $R$ be a semiprime *-ring having quasi-*-IFP. Set $a^{2}=a a^{*}=0$ for some $a \in R$, then $a R a=a R a^{*}=0$, from Proposition 1. Since $R$ is semiprime, then $a=0$ and $R$ is *-reduced.

Finally, one can easily show that the class of *-rings having quasi-*-IFP is closed under direct sums (with changeless involution) and under taking *-subrings.

Proposition 4. The class of *-rings having quasi-*-IFP is closed under direct sums and under taking $*_{\text {-subrings. }}$

\section{3. *-REVERSIBLE *-RINGS}

Definition 2. An ideal $I$ of a $*$-ring $R$ is called $*$ reversible if $a b, a b * \in I$ implies $b a \in I$, for every $a, b \in R$. 
It is obvious that if $I$ is *-reversible then $a b, a b^{*} \in I$ implies also $b^{*} a \in I$, for every $a, b \in R$.

We note the following:

- A one-sided *-reversible ideal must be two-sided ideal.

- The *-reversible ideal may not be self adjoint according to the following example.

Example 3. Let $R$ be the ${ }^{*}$-ring in Example 1. The ideal $I=\left(\begin{array}{cc}F & F \\ 0 & 0\end{array}\right)$ is *reversible but not self-adjoint

Definition 3. A *-ring $R$ is said to be *-reversible if 0 is a *-reversible ideal of $R$; that is $a b=a b^{*}=0$ implies $b a=0$ (consequently $b^{*} a=0$ ), for every $a, b \in R$.

Example 4. Every $*$-domain is a $*$-reversible $*$-ring.

It is clear that every reversible ring with involution is *-reversible. But the converse is not always true as shown by the next example.

Example 5. Let $R$ be the *-ring in Example 1. $R$ is not reversible since the matrices $\alpha=\left(\begin{array}{ll}0 & 1 \\ 0 & 0\end{array}\right)$ and $\beta=\left(\begin{array}{ll}1 & 1 \\ 0 & 0\end{array}\right)$ satisfy $\alpha \beta=0$ while $\beta \alpha \neq 0$. Moreover, it easy to check that $R$ is *-reversible.

The following are some equivalents for a $*$-ring to be $*$-reversible.

Proposition 5. For $a *$-ring $R$, the following statements are equivalent.

(i) $R$ is *-reversible.

(ii) $r_{*}(S)=l_{*}(S)$ for every subset $S$ of $R$.

(iii) $r_{*}(a)=l_{*}(a)$ for every element $a \in R$.

(iv) For any two nonempty subsets $A$ and $B$ of $R, A B=A B^{*}=0$ implies $B A=$ 0 (consequently $B^{*} A=0$ ).

Proof. $(i) \Rightarrow(i i)$ : Let $x \in r_{*}(S)$, then $s x=s x^{*}=0$ for every $s \in S$. Since $R$ is *-reversible, we have $x s=x^{*} s=0$ for every $s \in S$. Hence, $x S=x^{*} S$ implies $x \in$ $l_{*}(S)$ and we get $r_{*}(S) \subseteq l_{*}(S)$. Similarly, $l_{*}(S) \subseteq r_{*}(S)$ and $r_{*}(S)=l_{*}(S)$ follows.

(ii $) \Rightarrow($ i i i $)$ is direct by considering $S$ as the singleton set $\{a\}$.

(ii i ) $\Rightarrow\left(\right.$ iv): Set $A B=A B^{*}=0$ for some nonempty subsets $A$ and $B$ of $R$. Then $a b=a b^{*}=0$ for every $a \in A$ and $b \in B$, and hence $b \in r_{*}(a)=l_{*}(a)$ from the condition. Therefore $b a=b^{*} a=0=0$ for every $a \in A$ and $b \in B$ which implies $B A=B^{*} A=0$.

$(i v) \Rightarrow(i)$ is direct by considering $A$ and $B$ as the singleton sets containing $a$ and $b$, respectively.

The question when does a *-reversible *-ring become reversible has been answered in the following proposition. 
Proposition 6. Let $R$ be $a *_{\text {-reversible }}^{*}$-ring and either

(1) $R$ has *-IFP, or

(2) * is proper.

Then, $R$ is reversible.

Proof. (1) Let $R$ have *-IFP and $a b=0$ for some $a, b \in R$. Then, by [1, Proposition 7], $a R b^{*}=0$ and hence $a b^{*}=0$. The *-reversibility of $R$ implies $b a=0$ and $R$ is reversible.

(2) Let the involution * be proper and $a b=0$ for some $a, b \in R$. Then $a\left(b b^{*}\right)=$ $a\left(b b^{*}\right)^{*}=0$ and hence $b b^{*} a=0$ from the *-reversibility of $R$. Now $\left(a^{*} b\right)\left(a^{*} b\right)^{*}=a^{*} b b^{*} a=0$ implies $a^{*} b=b^{*} a=0$, since * is proper. Finally, by the *-reversibility of $R, b^{*} a a^{*}=0$ implies $a a^{*} b^{*}=0$ and $(b a)(b a)^{*}=b a a^{*} b^{*}=0$ implies $b a=0$. Hence $R$ is reversible.

Now, we see that each $*_{\text {-reversible }}^{*}$-ring has quasi-*-IFP.

Proposition 7. Every *-reversible *-ring has quasi-*-IFP.

Proof. Let $a b=a b^{*}=0$ for some elements $a, b$ of a *-reversible *-ring $R$. Using the *-reversibility of $R$, we have $b a=b^{*} a=0$ which implies $b a r=b^{*} a r=0$. Again, by the *-reversibility of $R, a r b=a r b^{*}=0$ for every $r \in R$. Therefore $a R b=a R b^{*}=0$ which means that $R$ has quasi-*-IFP, by Proposition 1 .

From Propositions 7 and 2, we get the following.

Corollary 1. Every *-reversible *-ring is *-Abelian.

However, the next example shows that the converse of the previous proposition and its corollary is not always true.

Example 6. Let $D$ be a commutative domain. Then the ring

$$
R=\left\{\left(\begin{array}{ccc}
a & b & d \\
0 & a & c \\
0 & 0 & a
\end{array}\right) \mid a, b, c, d \in D\right\}
$$

has IFP, by [11, Proposition 1.2]. Define an involution $*$ on $R$ as $\left(\begin{array}{ccc}a & b & d \\ 0 & a & c \\ 0 & 0 & a\end{array}\right)^{*}=\left(\begin{array}{ccc}a & c & -d \\ 0 & a & b \\ 0 & 0 & a\end{array}\right)$. One can easily check that $R$ has quasi-*-IFP and hence is *-Abelian. But $R$ is not *-reversible since the elements $\alpha=\left(\begin{array}{ccc}0 & 0 & 0 \\ 0 & 0 & 1 \\ 0 & 0 & 0\end{array}\right)$ and $\beta=\left(\begin{array}{ccc}0 & 1 & 0 \\ 0 & 0 & 0 \\ 0 & 0 & 0\end{array}\right)$ of $R$ satisfy $\alpha \beta=\alpha \beta^{*}=0$ but $\beta \alpha=\left(\begin{array}{ccc}0 & 0 & 1 \\ 0 & 0 & 0 \\ 0 & 0 & 0\end{array}\right) \neq 0$ 
Moreover, if the involution * is proper then the properties IFP, *-IFP, quasi-*-IFP, *-reversibility and reducedness are identical as shown in the following result.

Proposition 8. Let $R$ be $a *$-ring and the involution *is proper. Then the following conditions are equivalent:

(1) $R$ is *-reversible

(2) $R$ has quasi-*-IFP.

(3) $R$ has IFP.

(4) $R$ has *-IFP.

(5) $R$ is reduced.

Proof. (3),(4) and (5) are equivalent from [1, Proposition 9].

$(1) \Rightarrow(2)$ is direct from Proposition 7.

$(2) \Rightarrow(3)$ : Let $a b=0$ for some $a, b \in R$. Then $a\left(b b^{*}\right)=a\left(b b^{*}\right)^{*}=0$ implies $a R b b^{*}=0$ from the quasi-*-IFP of $R$. Now $(a r b)(a r b)^{*}=a r b b^{*} r^{*} a^{*}=0$ implies $a r b=0$ fore every $r \in R$ since * is proper. Therefore $a R b=0$ and so $R$ has IFP.

$(5) \Rightarrow(1)$ : Let $a b=a b^{*}=0$ for some $a, b \in R$, then $(b a)^{2}=b a b a=0$ and $\left(b^{*} a\right)^{2}=b^{*} a b^{*} a=0$. Hence, $b a=b^{*} a=0$ from the reducedness of $R$ and so $R$ is *-reversible.

Next, we discuss the converse of Example 4; that is when a $*$-reversible $*$-ring is *-domain.

Proposition 9. $A *$-ring is $a *$-domain if and only if $R$ is $*$-prime and $*$-reversible.

Proof. First, Suppose that $R$ is a *-domain, hence $R$ is obviously *-reversible. Let $I J=0$ for some *-ideals $I$ and $J$ of $R$, then $a b=a b^{*}=0$ for every $a \in I$ and $b \in J$. Hence, either $a=0$ or $b=0$ which implies $I=0$ or $J=0$ and so $R$ is *-prime. Conversely, let $R$ be both *-prime and *-reversible and $a b=a^{*} b=0$ for some $0 \neq a, b \in R$. We have $r^{*} b^{*} a^{*}=r^{*} b^{*} a=0$ for every $r \in R$ and so $a^{*} r^{*} b^{*}=a r^{*} b^{*}=0$ for every $r \in R$ from the *-reversibility of $R$, which gives $b R a=b R a^{*}=0$. Since $R$ is *-prime and $a \neq 0$, we get $b=0$, by [ [6], Proposition $5.4]$, and so $R$ has no *-zero divisors; that is a *-domain.

As a consequence, we get Proposition 4 in [3] as a corollary.

Corollary 2 ( [3], Proposition 4). If $R$ is a reduced ${ }^{*}$-prime ${ }^{*}$-ring, then $R$ is *-domain.

For a *-ring $R$, the trivial extension of $R$, denoted by $T(R, R)$, is the ring $\left\{\left(\begin{array}{cc}a & b \\ 0 & a\end{array}\right) \mid a, b \in R\right\}$. One can define the componentwise involution $\left(\begin{array}{cc}a & b \\ 0 & a\end{array}\right)^{*}=$ $\left(\begin{array}{cc}a^{*} & b^{*} \\ 0 & a^{*}\end{array}\right)$ to make $T(R, R)$ a *-ring.

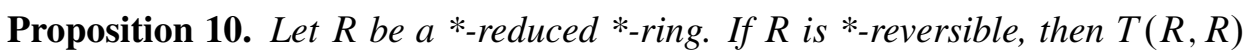
is a $*_{\text {-reversible }}^{*}$-ring. 
Proof. Let $\left(\begin{array}{ll}a & b \\ 0 & a\end{array}\right)\left(\begin{array}{ll}\alpha & \beta \\ 0 & \alpha\end{array}\right)=\left(\begin{array}{cc}a & b \\ 0 & a\end{array}\right)\left(\begin{array}{cc}\alpha^{*} & \beta^{*} \\ 0 & \alpha^{*}\end{array}\right)=\left(\begin{array}{ll}0 & 0 \\ 0 & 0\end{array}\right)$. Then $a \alpha=a \alpha^{*}=0$ and $a \beta+b \alpha=a \beta^{*}+b \alpha^{*}=0$. Since $R$ is *-reversible then $\alpha a=$ $\alpha^{*} a=0$. By the *-reversibility of $R$, it is easy to see that $a R \alpha=0$. Now $0=$ $a \beta+b \alpha=\alpha(a \beta+b \alpha)=\alpha b \alpha$ and $0=a \beta^{*}+b \alpha^{*}=a \beta^{*} \alpha+b \alpha^{*} \alpha=b \alpha^{*} \alpha$. Hence $(b \alpha)^{2}=b \alpha b \alpha=0$ and $(b \alpha)(b \alpha)^{*}=b \alpha \alpha^{*} b^{*}=0$. Then $b \alpha=0$ because $R$ is *reduced and therefore $a \beta=0$. Similarly, one can show that $b \alpha^{*}=0$ and $a \beta^{*}=0$. Using the *-reversibility of $R$ again we get $\alpha b=\alpha^{*} b=\beta a=\beta^{*} a=0$ which implies $\left(\begin{array}{ll}\alpha & \beta \\ 0 & \alpha\end{array}\right)\left(\begin{array}{ll}a & b \\ 0 & a\end{array}\right)=\left(\begin{array}{cc}\alpha^{*} & \beta^{*} \\ 0 & \alpha^{*}\end{array}\right)\left(\begin{array}{ll}a & b \\ 0 & a\end{array}\right)=\left(\begin{array}{ll}0 & 0 \\ 0 & 0\end{array}\right)$. Thus $T(R, R)$ is a *-reversible *-ring.

Furthermore, one can easily show that the class of *-reversible *-rings is closed under direct sums (using changeless involution) and taking *-subrings.

Proposition 11. The class of *-reversible *-rings is closed under direct sums and under taking $*_{- \text {subrings. }}$

\section{4. *-REFLEXIVE *-RINGS}

In this section, we introduce the involute version of reflexive ideals and rings defined by Mason [13] and study the relation between these rings and the *-reversible rings introduced in the previous section.

Definition 4. A ideal $I$ of a *-ring $R$ is called *-reflexive if for every $a, b \in R$, $a R b, a R b^{*} \subseteq I$ implies $b R a \subseteq I$ (consequently $b^{*} R a \subseteq I$ ). A *-ring $R$ is said to be *-reflexive if 0 is a *-reflexive ideal of $R$.

By the way, the ideal in the previous definition can not be one sided since for every $a \in I$ satisfying $a R \subseteq I$ implies $R a \subseteq I$ by taking $b=1$. Also, this ideal need not be self-adjoint by Example 3.

Example 7. Every *-reduced *-ring is *-reflexive.

It is evident that every reflexive *-ring is *-reflexive. However, the next example shows that the converse is not true.

Example 8. Let $D$ be a commutative domain and $R=\left\{\left(\begin{array}{ccc}\alpha & \beta & \delta \\ 0 & \alpha & \gamma \\ 0 & 0 & \alpha\end{array}\right) \mid \alpha, \beta, \gamma, \delta \in\right.$ $D\}$. $R$ is not reflexive according to [12, Example 2.3]. Define the involution $*$ : $\left(\begin{array}{lll}\alpha & \beta & \delta \\ 0 & \alpha & \gamma \\ 0 & 0 & \alpha\end{array}\right) \rightarrow\left(\begin{array}{ccc}\alpha & \gamma & \delta \\ 0 & \alpha & \beta \\ 0 & 0 & \alpha\end{array}\right)$. It is easy to check that $R$ is $*$-reversible and in particular is $*$-reflexive. 
Lemma 1. Let $R$ be a ring with semiproper involution *. Then $a R b=0$ implies $a R b^{*}=b R a=b^{*} R a=0$.

Proof.

$$
\begin{gathered}
\left(a r b^{*}\right) R\left(a r b^{*}\right)^{*}=a r b^{*} R b r^{*} a^{*} \subseteq a R b r^{*} a^{*}=0, \\
\text { for every } r \in R \text { implies } a R b^{*}=0,
\end{gathered}
$$

(bra) $R(b r a)^{*}=b r a R a^{*} r^{*} b^{*} \subseteq b r a R b^{*}=0$, for every $r \in R$ implies $b R a=0$ and

$\left(b^{*} r a\right) R\left(b^{*} r a\right)^{*}=b r a R a^{*} r^{*} b \subseteq b r a R b=0$, for every $r \in R$ implies $b^{*} R a=0$.

Corollary 3. Every *-ring with semiproper involution is reflexive (and hence $*_{-}$ reflexive).

The converse of the previous corollary is not necessary true as shown in the next example.

Example 9. If $F$ is a field, then the ring $R=F \oplus F^{o p}$, with the exchange involution $*$ defined by $(a, b)^{*}=(b, a)$ for all $a, b \in R$, is obviously a reflexive and hence $*$-reflexive but $*$ is not semiproper. Indeed, the element $0 \neq \alpha=(0, a)$ for some nonzero element $a$ of $F$ satisfies $\alpha R \alpha^{*}=0$.

In the following proposition, we state some equivalent definitions for a $*$-ring to be ${ }^{*}$-reflexive .

Proposition 12. For $a *_{-}$ring $R$, the following statements are equivalent :

(i) $R$ is *-reflexive.

(ii) $r_{*}(a R)=l_{*}(R a)$ for every $a \in R$.

(iii) For any two nonempty subsets $A$ and $B$ of $R, A R B=A R B^{*}=0$ implies $B R A=B^{*} R A=0$.

Proof. $(i) \Rightarrow(i i)$ : Let $x \in r_{*}(a R)$, then $a R x=a R x^{*}=0$. Hence $x R a=x^{*} R a=$ 0 , by the *-reflexivity of $R$, implies $x \in l_{*}(R a)$ and so $r_{*}(a R) \subseteq l_{*}(R a)$. Similarly, $l_{*}(a R) \subseteq r_{*}(R a)$ and we get $r_{*}(a R)=l_{*}(R a)$.

(ii $) \Rightarrow($ iii $)$ : Set $A R B=A R B^{*}=0$ for some subsets $A$ and $B$ of $R$. Then $a R b=a R b^{*}=0$ for every $a \in A$ and $b \in B$, and hence $b \in r_{*}(a R) \subseteq l_{*}(R a)$ from the condition. Therefore $b R a=b^{*} R a=0$ for every $a \in A$ and $b \in B$ which implies $B R A=b^{*} R A=0$.

(ii $i) \Rightarrow($ i $)$ is direct by considering $A$ and $B$ as the singleton sets containing $a$ and $b$, respectively..

The following proposition and example show that the class of *-reflexive *-rings generalizes strictly that of $*$-reversible *-rings. 
Proposition 13. Every $*_{\text {-reversible }} *_{\text {-ring }}$ is $*_{\text {-reflexive }}$

Proof. Let $a R b=a R b^{*}=0$, then $a b=a b^{*}=0$ implies $r a b=r a b^{*}=0$, for every $r \in R$. So that $b r a=b^{*} r a$ for every $r \in R$, from the *-reversibility of $R$. Thus $b R a=b^{*} R a=0$ and hence $R$ is *-reflexive.

Example 10. Let $n>2$ be an integer and $p \leq n$ be a prime number. The *-ring $R=\mathrm{M}_{n}\left(\mathbb{Z}_{p}\right)$, where $*$ is the transpose involution, is prime and hence reflexive (in particular *-reflexive). Moreover, $R$ is not*-reversible. Indeed, the nonzero elements

$$
\begin{aligned}
& \alpha=e_{12}+e_{13}+\cdots+e_{1 n}, \\
& \beta=e_{11}+e_{12}+\cdots+e_{1(n-1)}+2 e_{1 n}
\end{aligned}
$$

of $R$, where $e_{i j}$ is the square matrix of order $n$ with 1 in the $(i, j)$-position and 0 elsewhere, satisfy $\alpha \beta=\alpha \beta^{*}=0$, while $\beta \alpha \neq 0$ and $\beta^{*} \alpha \neq 0$.

The question when a *-reflexive *-ring is *-reversible is answered in the following proposition.

Proposition 14. A*-ring $R$ is *-reversible if and only if $R$ has quasi-*-IFP and *-reflexive.

Proof. The necessity is obvious. For sufficiency, let $a b=a b^{*}=0$ for some $a, b \in$ $R$. Since $R$ has quasi-*-IFP, then $a R b=a R b^{*}=0$. The *-reflexivity of $R$ implies $b R a=b^{*} R a=0$. Hence $b a=b^{*} a=0$ and $R$ is *-reversible.

In the next result we discuss when a principal right ideal generated by a projection in a $*$-reflexive $*$-ring is $*$-reflexive.

Proposition 15. Let e be a projection of a *-reflexive ${ }^{*}$-ring $R$. Then e is central if and only if $e R$ is a $*$-reflexive $*$-ideal.

Proof. Let $e$ be central and $a R b, a R b^{*} \subset e R$, then $a r b=e a r b$ and $a r b^{*}=$ $e a r b^{*}$ for every $r \in R$. Hence $(1-e) a R b=(1-e) a R b^{*}=0$ and consequently $(1-e) b R a=(1-e) b^{*} R a=0$, since $R$ is *-reflexive and $e$ is central. Hence $b R a, b^{*} R a \subseteq e R$ and $e R$ is *-reflexive ideal. The converse implication is clear since $e R$ is a ${ }^{*}$-ideal and so $e$ is central.

Now, we show that $*$-reflexive property is extended to the $*$-corner.

Proposition 16. Let $R$ be $a *_{\text {-reflexive }}^{*}$-ring, then the *-corner eRe for every projection e of $R$ is also *-reflexive.

Proof. Let $R$ be *-reflexive and $a=e x e, b=e y e \in e R e$ such that $a(e R e) b=$

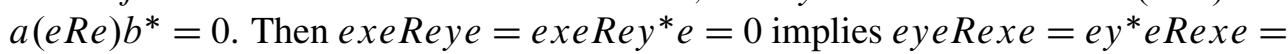
0 , since $R$ is *-reflexive. Therefore $b(e R e) a=b^{*}(e R e) a=0$ and so $e R e$ is *reflexive. 


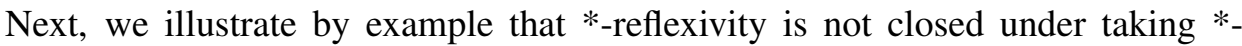
subrings.

Example 11. The ring $R=\mathrm{M}_{2}\left(\mathbb{Z}_{2}\right)$ is prime and hence reflexive. The upper triangular matrix ring $S=\left(\begin{array}{cc}\mathbb{Z}_{2} & \mathbb{Z}_{2} \\ 0 & \mathbb{Z}_{2}\end{array}\right)$ over $\mathbb{Z}_{2}$ is a *-subring of $R$ under the involution * defined as $\left(\begin{array}{ll}a & b \\ d & c\end{array}\right)^{*}=\left(\begin{array}{cc}c & -b \\ -d & a\end{array}\right) . R$ is clearly *-reflexive but $S$ is not, since the elements $\alpha=\left(\begin{array}{ll}0 & 0 \\ 0 & 1\end{array}\right)$ and $\beta=\left(\begin{array}{ll}0 & 1 \\ 0 & 0\end{array}\right)$ of $R$ satisfy $\alpha R \beta=\alpha R \beta^{*}=0$ but $\beta R \alpha=\beta^{*} R \alpha=\left(\begin{array}{cc}0 & \mathbb{Z}_{2} \\ 0 & 0\end{array}\right) \neq 0$

We end this section by showing that the *-reflexivity is restricted from the full matrix ring to its underlying ring.

Proposition 17. If $\mathrm{M}_{n}(R)$ is a *-reflexive *-ring for some $n \geq 1$ and with the transpose involution *, then $R$ is also a *-reflexive *-ring .

Proof. let $\mathrm{M}_{n}(R)$ be a *-reflexive *-ring for some $n \geq 1$. Since $R \cong e_{11} \mathrm{M}_{n}(R) e_{11}$, as *-rings, then $R$ is *-reflexive, by Proposition 16 .

\section{PROJECTION *-REFLEXIVE RINGS}

In this last section, we give another generalization for the class of *-reflexive rings; that is projection $*$-reflexive $*$-rings.

In [10], Kim defines an idempotent reflexive ring $R$ as the ring satisfying $a R e=0$ if and only if $e R a=0$ for every idempotent $e, a \in R$.

Definition 5. An ideal $I$ of a *-ring $R$ satisfies $a R e \subseteq I$ if and only if $e R a \subseteq I$ for every projection $e, a \in R$, is called projection *-reflexive. A *-ring $R$ is called projection *-reflexive if 0 is a projection *-reflexive ideal.

The ideal $I$ of the previous definition can not be one-sided ideal, because if $I$ is a right ideal then $a R 1 \subseteq I$ for every $a \in I$ implies $1 R a \subseteq I$, since 1 is a projection. Moreover, the ideal $I$ in the definition need not be self-adjoint; indeed, for a field $F$ the *-ring $F \bigoplus F$ with the exchange involution, possesses the non self-adjoint projection *-reflexive ideal $(0, F)$.

It is evident from the definition that *-reflexive and idempotent reflexive *-rings are projection *-reflexive. Accordingly, we raise the following two questions.

- Is there a projection *-reflexive *-ring which is not idempotent reflexive?

- Is there a projection *-reflexive *-ring which is not*-reflexive?

The answers of these questions are in the following example. 
Example 12. The *-ring $R=\left(\begin{array}{cc}F & F \\ 0 & F\end{array}\right)$ over a field $F$ with the involution * defined by $\left(\begin{array}{cc}a & b \\ 0 & c\end{array}\right)^{*}=\left(\begin{array}{cc}c & -b \\ 0 & a\end{array}\right)$, is projection *-reflexive because $\left(\begin{array}{ll}1 & 0 \\ 0 & 1\end{array}\right)$ and $\left(\begin{array}{ll}0 & 0 \\ 0 & 0\end{array}\right)$ are the only projections of $R$. Clearly, $R$ is not idempotent reflexive, since the idempotent $\left(\begin{array}{ll}1 & 0 \\ 0 & 0\end{array}\right)$ of $R$ satisfies

$$
\left(\begin{array}{ll}
0 & 2 \\
0 & 0
\end{array}\right)\left(\begin{array}{ll}
F & F \\
0 & F
\end{array}\right)\left(\begin{array}{ll}
1 & 0 \\
0 & 0
\end{array}\right)=\left(\begin{array}{ll}
0 & 0 \\
0 & 0
\end{array}\right)
$$

while

$$
\left(\begin{array}{ll}
1 & 0 \\
0 & 0
\end{array}\right)\left(\begin{array}{ll}
F & F \\
0 & F
\end{array}\right)\left(\begin{array}{ll}
0 & 2 \\
0 & 0
\end{array}\right)=\left(\begin{array}{ll}
0 & F \\
0 & 0
\end{array}\right) \neq 0 .
$$

Moreover, $R$ is not *-reflexive, since

$$
\begin{gathered}
\left(\begin{array}{ll}
0 & 1 \\
0 & 1
\end{array}\right)\left(\begin{array}{ll}
F & F \\
0 & F
\end{array}\right)\left(\begin{array}{ll}
0 & 1 \\
0 & 0
\end{array}\right)= \\
\left(\begin{array}{ll}
0 & 1 \\
0 & 1
\end{array}\right)\left(\begin{array}{cc}
F & F \\
0 & F
\end{array}\right)\left(\begin{array}{cc}
0 & -1 \\
0 & 0
\end{array}\right)=\left(\begin{array}{ll}
0 & 0 \\
0 & 0
\end{array}\right)
\end{gathered}
$$

while

$$
\left(\begin{array}{ll}
0 & 1 \\
0 & 0
\end{array}\right)\left(\begin{array}{ll}
F & F \\
0 & F
\end{array}\right)\left(\begin{array}{ll}
0 & 1 \\
0 & 1
\end{array}\right)=\left(\begin{array}{cc}
0 & F \\
0 & 0
\end{array}\right)
$$

The proof of the following proposition, which gives an equivalent definition for projection *-reflexive *-rings, is straightforward.

Proposition 18. $A *$-ring $R$ is projection *-reflexive if and only if for any nonempty subset $A$ and any projection e of $R, A R e=0$ implies $e R A=0$.

Obviously, every *-Abelian *-ring is projection *-reflexive and consequently every *-ring having quasi-*-IFP is also projection *-reflexive, by Proposition 2. However, the converse of this statement needs additional condition, as in the next proposition.

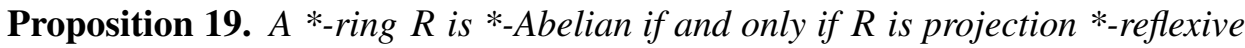
and satisfies $e R(1-e) R e=0$ for every projection e of $R$.

Proof. The necessity is obvious, For sufficiency, let $e$ be an arbitrary projection of the projection *-reflexive *-ring $R$ and $e R(1-e) R e=0$. By Proposition 18 , we have $e R e R(1-e)=0$ and taking involution gives $(1-e) R e R e=0$. Hence, $(1-e) R e=0$ which implies that $e$ is semicentral, from [Lemma 1.1, [8]], and hence it is central. Thus $R$ is *-Abelian

In the next result we show when a projection in a projection $*$-reflexive $*$-ring is central. 
Proposition 20. Let $R$ be a projection *-reflexive *-ring and $e$ is a projection of $R$. Then the following are equivalent:

(i) $e$ is central.

(ii) eR is a projection-*-reflexive $*_{\text {-ideal. }}$

Proof. (i) $\Rightarrow$ (ii): Assume that $a R f \subset e R$ for some projection $f$ of $R$. So that $\operatorname{arf}=e \operatorname{arf}$ for every $r \in R$ and hence $(1-e) a R f=0$. Therefore $f R(1-e) a=$ $0=(1-e) f R a$, since $R$ is projection *-reflexive, and consequently $f R a=e f R a \subseteq$ $e R$. Hence $e R$ is a projection-*-reflexive ideal.

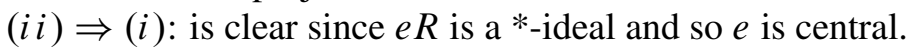

Corollary 4. If every principal *-ideal of $R$ is projection *-reflexive, then $R$ is *-Abelian.

Finally, Since the only projections of the *-corner $e$ Re is the projection $e$, then $e R e$ is projection *-reflexive if $R$ is projection *-reflexive.

Proposition 21. Let $R$ be a projection *-reflexive *-ring, then the *-corner eRe, for every projection e of $R$, is also projection *-reflexive.

$$
\text { 6. *-NILPOTENCY IN *-REVERSIBLE*-RINGS }
$$

According to [3], in a *-ring $R$ every *-nilpotent element is nilpotent but the converse is not always true as shown in [3, Example 2.2]. In the next, we give a sufficient condition that makes a nilpotent element *-nilpotent.

Proposition 22. In $a *$-reversible $*$-ring $R$, every nilpotent element is $*$-nilpotent.

Proof. Let $a$ be a nilpotent element of a ${ }^{*}$-reversible *-ring $R$. Hence $a^{n}=0$, for some positive integer $n$, and multiplying by $a^{*}$ form right, we get $a^{n-1}\left(a a^{*}\right)=0$. From the *-reversibility of $R$, we have $\left(a a^{*}\right) a^{n-1}=0$. Multiply again by $a^{*}$ form right and apply the *-reversible property, we get $\left(a a^{*}\right) a^{n-2}=0$. Continuing this process, we get $\left(a a^{*}\right)^{n}=0$ and $a$ is *-nilpotent.

However, the *-reversibility condition in the previous proposition is sufficient but not necessary as clear from Example 6 . Indeed, the elements of the $*_{\text {-ideal }}$ $\left(\begin{array}{ccc}0 & D & D \\ 0 & 0 & D \\ 0 & 0 & 0\end{array}\right)$ are precisely all the nilpotent (which also *-nilpotent) elements of the ring $R$.

Corollary 5. Every ${ }^{*}$-reduced $*_{\text {-reversible }}^{*}$-ring is reduced.

By the definition of nilpotency, an element is nilpotent if and only if a power of it is also nilpotent. This is not the case for *-nilpotent elements as shown in the following examples. 
Example 13. In the *-ring $R=\mathrm{M}_{2}(\mathbb{C})$ of $2 \times 2$ matrices with complex entries and transpose involution $*$, the element $a=\left(\begin{array}{cc}\frac{\sqrt{3}+l}{2} & 1 \\ 1 & \frac{\sqrt{3}-l}{2}\end{array}\right)$ satisfies $\left(a a^{*}\right)^{6}=$ $\left(\begin{array}{cc}-1 & 1 \\ 1 & -1\end{array}\right)$ which can not tend to zero ever with any power. Thus $a$ is not $*-$ nilpotent, while $\left(a^{3}\left(a^{3}\right)^{*}\right)^{1}=\left(a^{3}\right)^{2}=0$ which means that $a^{3}$ is *-nilpotent.

In the next, a sufficient condition is given to make *-nilpotency transfers between the element and its powers.

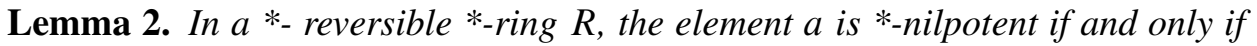
$a^{2}$ is *-nilpotent.

Proof. Let $a$ be a ${ }^{*}$-nilpotent element of $R$, then $a^{n}=\left(a a^{*}\right)^{m}=0$, for some positive integers $m$ and $n$. Now, $0=\left(a a^{*}\right)^{m}=a^{*}\left(a a^{*}\right)^{m}=a^{*}\left(a a^{*}\right)^{m-1}\left(a a^{*}\right)$ and from the *-reversibility of $R$, we get $0=\left(a a^{*}\right) a^{*}\left(a a^{*}\right)^{m-1}=a\left(a^{*}\right)^{2}\left(a a^{*}\right)^{m-1}$. Multiply the last equation by $a$ from right to get $a\left(a^{*}\right)^{2} a\left(a^{*} a\right)^{m-2}\left(a^{*} a\right)=0$ and applying the *-reversible property again, we get $a^{*} a^{2}\left(a^{*}\right)^{2} a\left(a^{*} a\right)^{m-2}=0=$ $a^{*} a^{2}\left(a^{*}\right)^{2}\left(a a^{*}\right)^{m-2} a$. Multiply again by $a^{*}$ from right and apply the *-reversibility, we get $a\left(a^{*}\right)^{2} a^{2}\left(a^{*}\right)^{2}\left(a a^{*}\right)^{m-2}=0$. Continuing, we get $\left(a^{2}\left(a^{*}\right)^{2}\right)^{m}=0$ and $a^{2}$ is *-nilpotent.

For sufficiency, if $a^{2}$ is *-nilpotent; that is $\left(a^{2}\right)^{n}=0=\left(a^{2}\left(a^{*}\right)^{2}\right)^{m}$ for some positive integers $m$ and $n$, we get by the same procedure as above $\left(a^{*} a\right)^{4} m=0$ and $a$ is *-nilpotent.

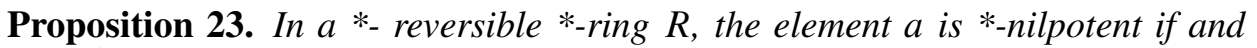
only if $a^{k}$ is also *-nilpotnet for every positive integer $k$.

Proof. The sufficient condition is clear. For the necessity, let $a$ be a *-nilpotent element of $R$, then $a^{l}=\left(a a^{*}\right)^{n}=0$ for some positive integers $l$ and $n$. We use induction on $k$ to show that $a^{k}\left(a^{*}\right)^{k}$ is nilpotent. The case $k=2$ is clear from Lemma 2. Now, we have to show that $a^{k+1}\left(a^{*}\right)^{k+1}$ is also nilpotent if $a^{k}\left(a^{*}\right)^{k}$ is nilpotent. Now, if $0=\left(a^{k}\left(a^{*}\right)^{k}\right)^{m}=a^{k}\left(a^{*}\right)^{k}\left(a^{k}\left(a^{*}\right)^{k}\right)^{m-1}$, multiply by $\left(a^{*}\right)^{k+1} a$ from left and apply the *-reversibility, we get $\left(a^{*}\right)^{k}\left(a^{k}\left(a^{*}\right)^{k}\right)^{m-1}\left(a^{*}\right)^{k+1} a^{k+1}=0$. Multiply by $a^{*}$ from left and take involution of both sides, we obtain $\left(a^{*}\right)^{k+1} a^{k+1}\left(a^{k}\left(a^{*}\right)^{k}\right)^{m-1} a^{k+1}=0 . \quad$ The *-reversibility of $R$ gives $a^{k}\left(a^{*}\right)^{k}\left(a^{k}\left(a^{*}\right)^{k}\right)^{m-2} a^{k+1}\left(a^{*}\right)^{k+1} a^{k+1}=0$. Multiplying by $\left(a^{*}\right)^{k+1} a$ from left gives $\left(a^{*}\right)^{k+1} a^{k+1}\left(a^{*}\right)^{k}\left(a^{k}\left(a^{*}\right)^{k}\right)^{m-2} a^{k+1}\left(a^{*}\right)^{k+1} a^{k+1}=0$ and the *-reversibility of $R$ gives $\left(a^{*}\right)^{k}\left(a^{k}\left(a^{*}\right)^{k}\right)^{m-2} a^{k+1}\left(\left(a^{*}\right)^{k+1} a^{k+1}\right)^{2}=0$. Multiply again by $\left(a^{*}\right)^{k+1}$, we get $\left(a^{*}\right)^{k}\left(a^{k}\left(a^{*}\right)^{k}\right)^{m-2}\left(a^{k+1}\left(a^{*}\right)^{k+1}\right)^{3}=0$. Continuing, we get $\left(a^{*}\right)^{k}\left(a^{k+1}\left(a^{*}\right)^{k+1}\right)^{2 m-1}=0$ and multiplication by $a^{k+1} a^{*}$ gives $\left(a^{k+1}\left(a^{*}\right)^{k+1}\right)^{2 m}=0$. 
Conjecture 1 (Köthe's conjecture). If a ring has a non-zero nil right ideal, then it has a nonzero nil ideal, is still unsolved.

In [9, Theorem 2.2], Cohn proved that for reversible rings, Köthe's conjecture has an affirmative solution. In the next, we have a strong affirmative solution for *-reversible *-rings.

Proposition 24. Every *-reversible *-ring which is not *-reduced, contains a nonzero nilpotent ideal.

Proof. If $R$ is not *-reduced and *-reversible *-ring, then $R$ contains a nonzero *nilpotent element, say $a$. So that $a^{m}=\left(a a^{*}\right)^{n}=0$, for some positive integers $m$ and $n$. If $n=1$, we have $a^{m}=a a^{*}=0$ which implies $r_{1} a^{m}=r_{1} a^{m-1} a^{*}=0$ for every $r_{1} \in R$. From the *-reversibility of $R$, we get $a r_{1} a^{m-1}=0$. Again $r_{2} a r_{1} a^{m-1}=$ $r_{2} a r_{1} a^{m-2} a^{*}=0$ implies $a r_{2} a r_{1} a^{m-2}=0$ for every $r_{1}, r_{2} \in R$. Continuing, we get $(\operatorname{RaR})^{m}=0$; that is the ideal generated by $a$ is a nonzero nilpotent ideal. If $n>1$, we have $a a^{*} \neq 0$. Since $\left(a a^{*}\right)^{n}=0$, then $r_{1}\left(a a^{*}\right)^{n}=0$ gives $\left(a a^{*}\right) r_{1}\left(a a^{*}\right)^{n-1}=0$ due to the self-adjointness of $a a^{*}$ and using the *-reversible property. As before, we get $\left(R a a^{*} R\right)^{n}=0$; that is the *-ideal generated by $a a^{*}$ is a nonzero nilpotent ideal.

Corollary 6. In a $*$-reversible $*_{\text {-ring }} R$, if $R$ has a non-zero nil right ideal, then it has a nonzero nil ideal.

Corollary 7. Each semiprime $*_{\text {-reversible }}^{*}$-ring is *-reduced.

\section{CONCLUSION}

We can now sate the following implications in the class of rings with involution.

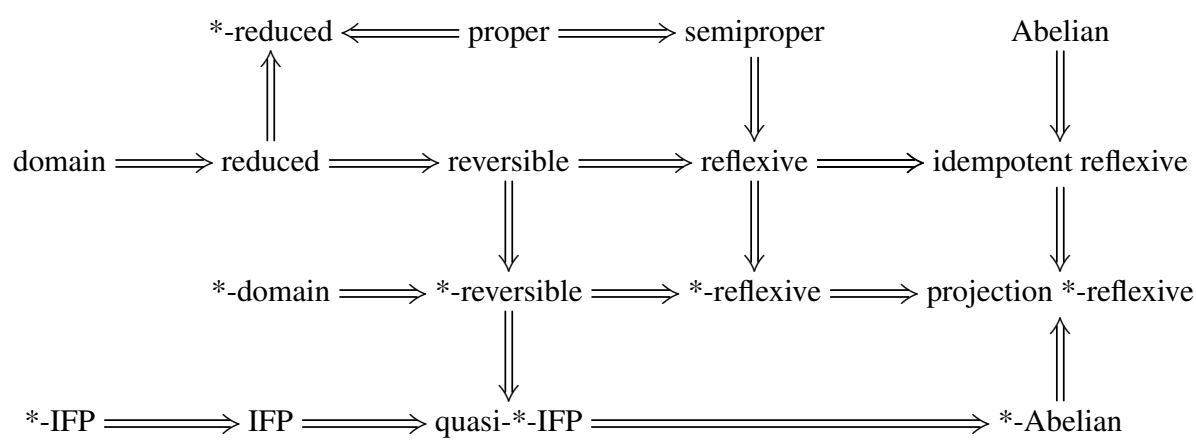

\section{REFERENCES}

[1] U. A. Aburawash and M. Saad, "On biregular, IFP and quasi-Baer *-rings," East-West J. Math., vol. 16, no. 2, pp. 182-192, 2014.

[2] U. A. Aburawash and K. B. Sola, “*-zero divisors and *-prime ideals,” East-West J. Math., vol. 12, no. 1, pp. 27-31, 2010. 
[3] U. A. Aburawash and M. Saad, “*-Baer property for rings with involution," Studia Sci. Math. Hungar, vol. 53, no. 2, pp. 243-255, 2016, doi: 10.1556/012.2016.53.2.1338.

[4] K. I. Beidar, L. Márki, R. Mlitz, and R. Wiegandt, "Primitive involution rings," Acta Mathematica Hungarica, vol. 109, no. 4, pp. 357-368, 2005, doi: 10.1007/S10474-005-0253-4.

[5] H. E. Bell, "Near-rings in which each element is a power of itself," Bull. Austral. Math. Soc., vol. 2, pp. 363-368, 1970, doi: 10.1017/S0004972700042052.

[6] G. F. Birkenmeier and N. J. Groenewald, "Prime ideals in rings with involution," Quaest. Math., vol. 20, no. 4, pp. 591-603, 1997, doi: 10.1080/16073606.1997.9632228.

[7] G. F. Birkenmeier, "Idempotents and completely semiprime ideals," Comm. Algebra, vol. 11, pp. 567-58, 1983, doi: 10.1080/00927878308822865.

[8] G. F. Birkenmeier, J. Y. Kim, and J. K. Park, "Quasi-Baer ring extensions and biregular rings," Bull. Austral. Math. Soc., vol. 61, no. 1, pp. 39-52, 2000, doi: 10.1017/S0004972700022000.

[9] P. M. Cohn, "Reversible rings," London Math. Soc., vol. 31, pp. 641-648, 1999, doi: 10.1112/S0024609399006116.

[10] J. Y. Kim and J. U. Baik, "On idempotent reflexive rings," Kyungpook Math. J., vol. 46, pp. 597$601,2006$.

[11] N. K. Kima and Y. Lee, "Extensions of reversible rings," J. Pure App. Algebra, vol. 185, pp. 207-223, 2003, doi: 10.1016/S0022-4049(03)00109-9.

[12] T. K. Kwak and Y. Lee, "Reflexive property of rings," Comm. Algebra, vol. 40, no. 4, pp. 15761594, 2012, doi: 10.1080/00927872.2011.554474.

[13] G. Mason, "Reflexive ideals," Comm. Algebra, vol. 9, pp. 1709-1724, 1981, doi: 10.1080/00927878108822678.

Authors' addresses

Usama A. Aburawash

Alexandria University, Department of Mathematics and Computer Science, Alexandria, Egypt

E-mail address: aburawshealexu.edu.eg

\section{Muhammad Saad}

Alexandria University, Department of Mathematics and Computer Science, Alexandria, Egypt

E-mail address: muhammad. saadealex-sci.edu.eg 